\title{
The Formation and Evolution of LMC Globular Clusters: The Database
}

\author{
S.F. Beaulieu, R. Elson, G. Gilmore, R.A. Johnson, N. Tanvir \\ Institute of Astronomy, University of Cambridge, Madingley Road, \\ Cambridge, CB3 OHA, UK \\ B. Santiago \\ Instituto de Fisica, Universidade Federal do Rio Grande do Sul, Av. \\ Bento Goncalves 9500, Porto Alegre RS, CEP: 91501-970, Brazil
}

\begin{abstract}
We present details of the database from a large Cycle 7 HST project to study the formation and evolution of rich star clusters in the LMC (see Elson et al., this volume). Our data set, which includes NICMOS, WFPC2 and STIS images of 8 clusters, will enable us to derive deep luminosity functions for the clusters and to investigate the universality of the stellar IMF. We will look for age spreads in the youngest clusters, quantify the population of binary stars in the cores of the clusters and at the half-mass radii, and follow the development of mass segregation.
\end{abstract}

\section{Introduction}

The Large Magellanic Cloud (LMC) is unique in containing massive star clusters at all stages of evolution. We are presently obtaining very deep HST images of 8 optimally selected clusters. This large observing program will enable us to probe the cluster's stellar content down to $0.2 \mathrm{M}_{\odot}$. In Cycle 7 , a total of 95 orbits have been granted for this project. We are using WFPC2 (F555W,F814W), NICMOS (all three cameras) (F110W,F160W) and STIS (CCD/F28X50LP) instruments in primary mode for each cluster, with parallel observing of surrounding fields as well, giving a total of 285 orbits of imaging data. These observations, in conjunction with state-of-the-art N-body models being calculated by the Institute of Astronomy's N-body group, will quantify the origin and evolution of rich star clusters in the Milky Way, the LMC and beyond.

\section{The LMC clusters}

Our clusters are grouped in four pairs, with ages of $10^{7}-10^{10} \mathrm{yr}$ (see Table 1) and are observed at their core and half-mass radius. They are among the richest clusters in the LMC and have masses $\approx 10^{4} \mathrm{M}_{\odot}$. In the youngest clusters, the fraction of primordial binaries, the stellar initial mass function (IMF), and the degree of primordial mass segregation will allow us to investigate the process of star formation in a protocluster. Age spreads and a search for pre-MS stars will reveal both the timescale for star formation, which has important implications 
Table 1. The LMC globular cluster sample

\begin{tabular}{lccccl}
\hline Cluster & $\begin{array}{c}\log a g e \\
(\mathrm{yr})\end{array}$ & {$[\mathrm{Fe} / \mathrm{H}]^{a}$} & $\begin{array}{l}\mathrm{R}_{g}{ }^{b} \\
\left({ }^{\circ}\right)\end{array}$ & $\begin{array}{l}\mathrm{R}_{c}{ }^{c} \\
(\prime \prime)\end{array}$ & Recent CMD \\
\hline NGC 1805 & 7.2 & $\ldots$ & 3.7 & $\ldots$ & $\ldots$ \\
NGC 1818 & 7.3 & -0.8 & 3.4 & 7.9 & Will et al. (1995) \\
NGC 1831 & 8.7 & -0.3 & 4.6 & 14.2 & Mateo (1998) \\
NGC 1868 & 8.8 & -0.6 & 5.4 & 5.1 & Corsi et al. (1994) \\
NGC 2209 & 9.1 & -1.0 & 5.7 & 20.0 & Corsi et al. (1994) \\
H14=SL506 & 9.2 & $\ldots$ & 4.4 & 10.3 & none available \\
NGC 2210 & 10.1 & -2.2 & 5.0 & 7.1 & Brocato et al. (1996) \\
H11=SL868 & 10.1 & -2.1 & 5.2 & 19.8 & Mighell et al. (1996) \\
\hline \hline
\end{tabular}

\footnotetext{
${ }^{a}$ The values of metallicity are quite uncertain; for many of the clusters widely differing values are quoted in the literature

${ }^{b}$ Distance from the LMC centre

${ }^{c}$ Core radius
}

for its trigger, and whether the low and high mass stars form sequentially or together. In the intermediate and old clusters, we will trace the development of mass segregation and the binary fraction in the core and at the half-mass radius; binaries play a crucial role in the structural evolution of a cluster, and in particular, affect the onset of core collapse. A comparison of the IMFs in our clusters will help answer the far-reaching question of whether there is such a thing as a universal IMF.

In addition to the $8 \mathrm{LMC}$ clusters, we are observing two LMC background fields in order to correct for contamination from LMC field stars and unresolved background galaxies. Also from these background fields and the parallel data, we will study the LMC field star population and the star formation history. We are also observing one galactic globular cluster of similar metallicity to the LMC clusters, NGC 6553, in order to provide an empirical calibration of the NICMOS and STIS passbands relative to absolute $\mathrm{V}$ magnitude and mass. We have similar data for 47 Tuc from the HST project 7419 (PI: R.F.G. Wyse).

The data reduction and analysis is performed using STSDAS and photometry is performed using the DAOPHOT package. Some preliminary results can be found in Elson et al. (this volume), Johnson et al. (1998), and at our webpage: http://www.ast.cam.ac.uk/LMC.

\section{References}

Brocato, E., et al. 1996, MNRAS, 282, 614

Corsi, C.E., et al. 1994, MNRAS, 271, 385

Mighell, K.J., et al. 1996, AJ, 111, 2314

Will, J.-M, Bomans, D.J., De Boer, K.S. 1995, A\&A, 295, 54

Johnson, R.A., et al. 1998, in NICMOS and the VLT, edited by Wolfram Freudling \& Richard Hook, ESO Conference \& Workshop Proceedings No 55, 140 